\title{
A 2-arc Transitive Hexavalent Nonnormal Cayley Graph on $\mathbf{A}_{119}$
}

\author{
Bo Ling ${ }^{1}(1)$, Wanting $\mathrm{Li}^{1}$ and Bengong Lou ${ }^{2, *}$ \\ 1 School of Mathematics and Computer Sciences, Yunnan Minzu University, Kunming 650031, China; \\ boling@ynni.edu.cn (B.L.); wantingli08@163.com (W.L.) \\ 2 School of Mathematics and Statistics, Yunnan University, Kunmin 650031, China \\ * Correspondence: bglou@ynu.edu.cn
}

check for

updates

Citation: Ling, B.; Li, W.; Lou, B. A 2-arc Transitive Hexavalent Nonnormal Cayley Graph on $\mathrm{A}_{119}$. Mathematics 2021, 9, 2935. https:/ / doi.org/10.3390/math9222935

Academic Editor: Seok-Zun Song

Received: 19 October 2021

Accepted: 16 November 2021

Published: 18 November 2021

Publisher's Note: MDPI stays neutral with regard to jurisdictional claims in published maps and institutional affiliations.

Copyright: (c) 2021 by the authors. Licensee MDPI, Basel, Switzerland. This article is an open access article distributed under the terms and conditions of the Creative Commons Attribution (CC BY) license (https:// creativecommons.org/licenses/by/ $4.0 /)$.

\begin{abstract}
A Cayley graph $\Gamma=\operatorname{Cay}(G, S)$ is said to be normal if the base group $G$ is normal in Aut $\Gamma$. The concept of the normality of Cayley graphs was first proposed by M.Y. Xu in 1998 and it plays a vital role in determining the full automorphism groups of Cayley graphs. In this paper, we construct an example of a 2-arc transitive hexavalent nonnormal Cayley graph on the alternating group $\mathrm{A}_{119}$. Furthermore, we determine the full automorphism group of this graph and show that it is isomorphic to $A_{120}$.
\end{abstract}

Keywords: simple group; nonnormal Cayley graph; arc-transitive graph; automorphism group

\section{Introduction}

Throughout this paper, all graphs are assumed to be finite and undirected.

For a graph $\Gamma$, we use $V \Gamma, E \Gamma, A r c \Gamma$ and Aut $\Gamma$ to denote the vertex set, edge set, arc set and full automorphism group of the graph $\Gamma$, respectively. A graph $\Gamma$ is said to be arc-transitive if the full automorphism group Aut $\Gamma$ acts transitively on $A r c \Gamma$. We use val $\Gamma$ to denote the valency of the $\Gamma$, and we say $\Gamma$ is a cubic, tetravalent, pentavalent or hexavalent graph, meaning val $\Gamma=3,4,5$ or 6 .

Let $G$ be a finite group with identity element 1 and $S$ (say Cayley subset) a subset of G such that $1 \notin S$ and $S=S^{-1}:=\left\{x^{-1} \mid x \in S\right\}$. Define the Cayley graph Cay $(G, S)$, that is, the Cayley graph of $G$ with respect to the Cayley subset $S$ as the graph with vertex set $G$ such that $g, h \in G$ are adjacent if and only if $h g^{-1} \in S$. It is easy to see that the valency of Cay $(G, S)$ is $|S|$. As we all know, $\operatorname{Cay}(G, S)$ is connected if and only if $\langle S\rangle=G$. On the other hand, letting $R(G)$ be the right regular representation of $G$ and letting AutCay $(G, S)$ be the full automorphism group of $\operatorname{Cay}(G, S)$, there are clearly $R(G) \leq \operatorname{AutCay}(G, S)$, and $R(G)$ acts transitively on the vertices of $\operatorname{Cay}(G, S)$. Then, the graph $\operatorname{Cay}(G, S)$ is vertex-transitive, and $G$ (or $R(G)$ ) can be viewed as a regular subgroup of AutCay $(G, S)$. Conversely, a connected graph $\Gamma$ is isomorphic to a Cayley graph of a group $G$ if and only if the full automorphism group Aut $\Gamma$ contains a subgroup which acts regularly on $V \Gamma$ and the subgroup is isomorphic to $G$ (see [1]). A Cayley graph $\Gamma=\operatorname{Cay}(G, S)$ is said to be a normal Cayley graph if the base group $G$ is normal in Aut $\Gamma$; otherwise, $\Gamma$ is said to be a nonnormal Cayley graph (see [2]).

The study about Cayley graphs on finite non-abelian simple groups has always attracted much attention because of Cayley graphs with high levels of symmetry; for example, vertex-transitivity, edge-transitivity and arc-transitivity are widely used in the design of interconnection networks. For more detailed applications, we recommend that readers refer to $[3,4]$. Let $G$ be a finite non-abelian simple group, and let $\Gamma=\operatorname{Cay}(G, S)$ be a connected arc-transitive Cayley graph on $G$. The main motivation for classifying 2-arc-transitive nonnormal Cayley graphs comes from the fact that Fang, Ma and Wang [5] proved all but finitely that many locally primitive Cayley graphs of valency $d \leq 20$ or a prime number of the finite non-abelian simple groups are normal. In [5] (Problem 1.2), they proposed the following problem: classify nonnormal locally primitive Cayley graphs (note 
that 2-arc-transitive graphs must be locally primitive) of finite simple groups with valency $d \leq 20$ or a prime number. To solve this problem, we should study each valency $d \leq 20$ or a prime number. In the case where $\Gamma$ is a cubic graph (3-valent), Li [6] proved that $\Gamma$ must be normal, except for seven exceptions. On the basis of Li's result, Xu et al. $[7,8]$ proved that $\Gamma$ must be normal, except for two exceptions on $A_{47}$. In the case where $\Gamma$ is a tetravalent graph (4-valent), Fang et al. in [9] proved that most of such $\Gamma$ are normal, except for Cayley graphs on a list of G. Further, Fang et al. in [10] proved that $\Gamma$ are normal when $\Gamma$ is 2-transitive, except for two graphs on $\mathrm{M}_{11}$. In the case where $\Gamma$ is a pentavalent graph (5-valent), Zhou and Feng [11] proved that all 1-transitive Cayley $\Gamma$ of simple groups are normal. Ling and Lou in [12] gave an example of a 2-transitive pentavalent nonnormal Cayley graph on $A_{39}$ Therefore, the next natural problem is to study the case of the 6-valent. However, there are no known nonnormal examples of hexavalent 2-arc-transitive Cayley graphs on finite simple groups.

The aim of this paper is to construct a nonnormal example of a connected 2-arc transitive hexavalent Cayley graph on a finite non-abelian simple group. Our main result is the following theorem.

Theorem 1. There exists a nonnormal example of a connected 2-arc-transitive hexavalent Cayley graph on the alternating group $\mathrm{A}_{119}$, and the full automorphism group of this graph is isomorphic to the alternating group $\mathrm{A}_{120}$.

\section{Preliminaries}

In this section, we give some necessary preliminary results which are used in later discussions.

Let $G$ be a finite group and let $H$ be a subgroup of $G$. Then we have the following result (see [13] (Ch. I, 1.4)).

Lemma 1. Let $G$ be a group and let $H$ be a subgroup of $G$. Let $\mathrm{N}_{G}(H)$ be the normalizer of $H$ in $G$, and let $\mathrm{C}_{G}(H)$ be the centralizer of $H$ in $G$. Then, $\mathrm{N}_{G}(H) / \mathrm{C}_{G}(H)$ is isomorphic to a subgroup of the automorphism group $\operatorname{Aut}(H)$ of $H$.

We next introduce the definition of a Sabidussi coset graph. Let $G$ be a group, $g \in G \backslash H$ such that $g^{2} \in H$, and let $H$ be a core-free subgroup of $G$. Define the Sabidussi coset graph $\operatorname{Cos}(G, H, g)$ of $G$ with respect to the core-free subgroup $H$ as the graph with vertex set $[G: H]$ (the set of cosets of $H$ in $G$ ) such that $H x$ and $H y$ are adjacent if and only if $y x^{-1} \in H g H$. The following lemma follows from [14], and it can be easily proved by the definition of the coset graphs (see [15] (Theorem 3) for example).

Lemma 2. Let $G$ be a group, $g \in G \backslash H$ such that $g^{2} \in H$, and let $H$ be a core-free subgroup of $G$. Let $\Gamma=\operatorname{Cos}(G, H, g)$ be a Sabidussi coset graph of $G$ with respect to $H$. Then, $\Gamma$ is G-arc-transitive and the following holds:

(1) The valency of the graph $\Gamma$ is equal to $\left|H: H \cap H^{g}\right|$.

(2) $\Gamma$ is a connected graph if and only if $\langle H, g\rangle=G$.

(3) If $G$ contains a subgroup $R$ is regular on $V \Gamma$, then $\Gamma \cong \operatorname{Cay}(R, S)$, where $S=R \cap H g H$.

Conversely, if $\Sigma$ is an $X$-arc-transitive graph, then $\Sigma$ is isomorphic to a Sabidussi coset graph $\operatorname{Cos}\left(X, X_{v}, g\right)$, where $g \in \mathrm{N}_{X}\left(X_{v w}\right)$ is a 2-element such that $g^{2} \in X_{v}$, and $v \in V \Sigma, w \in \Sigma(v)$.

Proof. Let $\Sigma$ be an $X$-arc-transitive graph. Let $v \in V \Sigma$ be a vertex of $\Sigma$ and $w \in \Sigma(v)$. Since $\Sigma$ is $X$-arc-transitive, there is $g$ such that $v^{g}=w$. For each $x \in X$, define $\varphi: H x \longrightarrow v^{x}$. Then we can verify that $\varphi$ is a graph isomorphic from $\Sigma$ to $\operatorname{Cos}\left(X, X_{v}, g\right)$. Since $\Sigma$ is undirected, we have $g^{2} \in X_{v}$. Hence, $(H \cap H g)^{g}=H \cap H g$. Thus, we can choose a 2-element $g$ satisfying $g \in \mathrm{N}_{X}\left(X_{v w}\right)$.

Let $t_{1} \geq 0$ and $t_{2} \geq 0$ be two integers. We denote by the $\{2,3\}$-group the finite group of the order $2^{t_{1}} 3^{t_{2}}$. Following the definition of relevant objects in [16] (Theorem 3.1), we 
have the following lemma, which is about the stabilizers of arc-transitive hexavalent graphs. For the structure of the received stabilizers, see the proof in [16] (Page 926).

Lemma 3. Let $s$ be a positive integer, and let $\Gamma$ be a connected hexavalent $(G, s)$-transitive graph for some $G \leq$ Aut $\Gamma$. Let $v \in V \Gamma$. Then $s \leq 4$ and one of the following statements holds:

(1) For $s=1$, the stabilizer $G_{v}$ is a $\{2,3\}$-group.

(2) For $s=2$, the stabilizer $G_{v} \cong \operatorname{PSL}(2,5), \operatorname{PGL}(2,5), \mathrm{A}_{6}$ or $\mathrm{S}_{6}$.

(3) For $s=3$, the stabilizer $G_{v} \cong \mathrm{D}_{10} \times \operatorname{PSL}(2,5), F_{20} \times \operatorname{PGL}(2,5), \mathrm{A}_{5} \times \mathrm{A}_{6}, \mathrm{~S}_{5} \times \mathrm{S}_{6} .\left(\mathrm{D}_{10} \times\right.$ $\operatorname{PSL}(2,5)) \cdot \mathbb{Z}_{2}$ with $\mathrm{D}_{10} \cdot \mathbb{Z}_{2}=F_{20}$ and $\operatorname{PSL}(2,5) \cdot \mathbb{Z}_{2}=\operatorname{PGL}(2,5)$, or $\left(\mathrm{A}_{5} \times \mathrm{A}_{6}\right) \rtimes \mathbb{Z}_{2}$ with $\mathrm{A}_{5} \rtimes \mathbb{Z}_{2}=\mathrm{S}_{5}$ and $\mathrm{A}_{6} \rtimes \mathbb{Z}_{2}=\mathrm{S}_{6}$.

(4) For $s=4$, the stabilizer $G_{v} \cong \mathbb{Z}_{5}^{2} \rtimes \operatorname{GL}(2,5)=\operatorname{AGL}(2,5)$.

\section{A 2-arc Transitive Hexavalent Nonnormal Cayley Graph on $\mathbf{A}_{119}$}

In this section, we construct a connected 2-arc transitive hexavalent nonnormal Cayley graph on $A_{119}$ and determine its full automorphism group. In fact, if $\Gamma:=\operatorname{Cay}(G, S)$ is a Cayley graph of a non-abelian simple group $G$, then $G$ is core free in $X$, where $G \leq X \leq$ Aut $\Gamma$. Let $v \in V \Gamma$ and $H=X_{v}$. Suppose that $|H|=n$. Then by Lemma 3, $n$ may be 60, 120, etc. Consider the action of $X$ on the set of $[X: G]$ by right multiplication; then, $X \lesssim S_{n}$. So, we may construct the nonnormal Cayley graph in $S_{n}$, where $n=60,120$, etc. The following example is really the case where we construct $n=120$.

Construction 1. Let $G$ be the alternating group on the set $\{2,3, \ldots, 120\}$. Then, $G \cong A_{119}$. Let $H=\langle a, b\rangle\left\langle X:=\mathrm{A}_{120}\right.$ (the alternating group on $\{1,2, \ldots, 120\}$ ), where the following holds:

$$
\begin{aligned}
a= & (1243)(5131217)(6141118)(7151019)(816920)(216110181) \\
& (226210282)(236310383)(246410484)(256510585)(266610686) \\
& (276710787)(2868108 \text { 88)(29 69 109 89)(30 70 110 90)(31 71 111 91) } \\
& (327211292)(337311393)(347411494)(357511595)(367611696) \\
& (377711797)(387811898)(397911999)(4080120100)(41565946) \\
& (42555748)(43546045)(44535847)(49515250), \\
b= & (12141)(22242)(32343)(42444)(52545)(62646)(72747) \\
& (82848)(92949)(103050)(113151)(123252)(133353)(143454) \\
& (153555)(163656)(173757)(183858)(193959)(204060)(6185110) \\
& (6286112)(6387109)(6488111)(6595106)(6693108)(6796105) \\
& (6894107)(6981113)(7082114)(7183115)(7284116)(73100119) \\
& (7499117)(7598120)(7697118)(7791101)(7889102)(7992103) \\
& (8090104) .
\end{aligned}
$$

Take $x \in X$ as follows:

$$
\begin{aligned}
& x=(179)(2 \text { 80)(3 60)(4 58)(5 113)(6 64)(7 114)(8 63)(9 112)(10 111)(11 12) } \\
& (13 \text { 47)(15 73)(16 106)(19 43)(20 41)(21 118)(22 120)(23 24)(25 50)(26 49) } \\
& \text { (27 68)(28 66)(30 62)(32 61)(33 42)(34 44)(35 103)(36 101)(37 107)(38 45) } \\
& \text { (40 75)(48 108)(53 54)(55 115)(56 116)(57 119)(59 117)(65 109)(67 110) } \\
& \text { (69 102)(70 104)(71 72)(76 105)(81 89)(82 93)(84 99)(85 98)(87 91)(88 94) } \\
& \text { (90 100)(96 97). }
\end{aligned}
$$

Define $\Sigma=\operatorname{Cos}(X, H, x)$. 
Lemma 4. The graph $\Sigma=\operatorname{Cos}(X, H, x)$ in Construction 1 is a connected 2-arc-transitive graph and isomorphic to the nonnormal hexavalent Cayley graph $\mathrm{Cay}(G, S)$ of $G$, determined by $S=$ $\left\{x_{1}, x_{1}^{-1}, x_{2}, x_{3}, x_{4}, x_{5}\right\}$ with the following:

$$
\begin{aligned}
x_{1}= & (2338369510145608077)(437971034635961075878) \\
& (5901066651281610011374)(618647698109146585105) \\
& (79211473684952262715)(82031416375598311740) \\
& (98810411699312054218111523569111171) \\
& (108711624558911853228210212709411272) \\
& (133430176244476786110)(1932)(2942489911939578410833) \\
& (4361), \\
x_{2}= & (297)(499)(573)(674)(78)(941)(1088)(1178)(1358)(1496) \\
& (1560)(1695)(1766)(1865)(1952)(2050)(2240)(2327)(2432)(2529) \\
& (2633)(2839)(3136)(3538)(43116)(4477)(4562)(4648)(4761)(49107) \\
& (51105)(53110)(5475)(55109)(5676)(63120)(64119)(6791)(6892) \\
& (69103)(7094)(71104)(7293)(79113)(8086)(81112)(83111)(87114) \\
& (8990)(98117)(100118)(101102), \\
x_{3}= & (339)(437)(5106)(6105)(750)(849)(935)(1045)(1136) \\
& (1247)(13113)(14114)(1516)(18118)(1928)(2056)(2192)(2391) \\
& (26120)(2794)(2930)(31107)(32108)(33112)(34110)(3897)(4098) \\
& (4142)(43102)(44101)(5187)(5285)(53117)(5496)(5789)(5890) \\
& (59116)(60115)(6280)(6367)(6472)(6569)(6673)(6879)(7176) \\
& (7578)(8182)(83109)(84111)(93119)(99104)(100103) \\
x_{4}= & (220)(37)(412)(59)(613)(819)(1116)(1518)(2149)(2384) \\
& (24101)(25112)(2628)(27110)(2994)(3060)(3196)(3259)(3385) \\
& (34120)(3587)(36118)(3853)(4055)(4276)(4474)(45119)(46117) \\
& (4748)(5064)(51102)(5467)(5665)(57108)(58106)(62104)(6382) \\
& (66116)(68114)(6986)(7188)(7397)(7599)(77105)(7880)(79107) \\
& (81103)(8991)(90115)(92113)(98109)(100111) \\
x_{5}= & (2111)(389)(432)(521)(6118)(723)(8117)(995)(1193) \\
& (1399)(1427)(15100)(1628)(17106)(1820)(19105)(2273)(2475) \\
& (29112)(3069)(33108)(3480)(35107)(3679)(3738)(39101)(40103) \\
& (4149)(4253)(4459)(4558)(4751)(4854)(5060)(5657)(61116) \\
& (6264)(63114)(6594)(6696)(67102)(68104)(7190)(72110)(7784) \\
& (7882)(85120)(86119)(8788)(91109)(97113)(98115) . \\
&
\end{aligned}
$$

Proof. Let $\Delta:=\{1,2, \ldots, 120\}$. Then, $X$ has a natural action on $\Delta$. By Magma [17], $\langle H, x\rangle=X$, and so the graph $\Sigma$ is connected by Lemma 2 (2). Furthermore, by Magma [17], we have that $H$ is regular on $\Delta$. However, $G$ is the stabilizer of point 1 in $X$. Hence, $X$ has a factorization $X=G H=H G$ with $G \cap H=1$. Therefore, $G$ is regular on $[X: H]$. By Lemma 2 (3), $\Sigma$ is isomorphic to a Cayley graph of $G=A_{119}$. Additionally, by the computation of Magma [17] (for the Magma code, see Appendix A), we have $\frac{|H|}{\left|H \cap H^{x}\right|}=6$. Hence, Lemma 2 (1) implies that $\Sigma$ is a hexavalent graph. Since $H \cong \operatorname{PGL}(2,5)$, Lemma 3 implies that $\Sigma$ is 2-arc transitive. Since $X$ is a non-abelian simple group, $G$ is not normal in $X \leq$ Aut $\Sigma$. It follows that $\Sigma$ is nonnormal. Let $x_{1}, x_{2}, x_{3}, x_{4}, x_{5}$ and $S$ be defined as in this lemma. By the computation of Magma [17] (for the Magma code, see Appendix B), we have $G \cap(H x H)=S$. Thus, by Lemma 2 (3), we have that $\Sigma$ is isomorphic to Cay $(G, S)$. This completes the proof of the lemma.

In the next lemma, we show that the full automorphism group $A u t \Sigma$ is isomorphic to alternating group $A_{120}$.

Lemma 5. The full automorphism group Aut $\Sigma$ of the 2-arc-transitive hexavalent graph $\Sigma=$ $\operatorname{Cos}(X, H, x)$ in Construction 1 is isomorphic to alternating group $\mathrm{A}_{120}$. 
Proof. Let $A=A u t \Sigma$. Assume first that the full automorphism group $A$ is quasiprimitive on $V \Sigma$. Let $N$ be a minimal normal subgroup of A. Then, $N$ is transitive on $V \Sigma$. It implies that $N$ is insoluble. Thus, $N$ is isomorphic to $T_{1} \times T_{2} \times \cdots \times T_{d}=T^{d}$, where $T_{i} \cong T$ for each $1 \leq i \leq d, T$ is a non-abelian simple group, and $d \geq 1$. Let $p$ be the largest prime factor of the order of $\mathrm{A}_{119}$. Then, $p>5$ and $p^{2} \wedge\left|\mathrm{A}_{119}\right|$. Since $N$ is transitive on $V \Sigma$ and $|V \Sigma|=\left|\mathrm{A}_{119}\right|$, we have that $p$ divides $|N|$. Assume that $d \geq 2$. Then, $p^{d}$ divides $|N|$. However, by Lemma 3 , the order of the stabilizer $\mathrm{A}_{v}$ divides $2^{7} \cdot 3^{3} \cdot 5^{3}$, and so $|\mathrm{A}|$ divides $2^{7} \cdot 3^{3} \cdot 5^{3} \cdot\left|\mathrm{A}_{119}\right|$ which is divisible by $p^{d}$, a contradiction. Hence, we have $d=1$ and $N=T \unlhd \mathrm{A}$. Let $C=\mathrm{C}_{\mathrm{A}}(T)$ be the centralizer of $T$ in $\mathrm{A}$. Then, $C \unlhd N_{\mathrm{A}}(T)=\mathrm{A}$ and $C T=C \times T$. If $C \neq 1$, since $A$ is quasiprimitive on $V \Sigma$, this implies that $C$ is transitive on $V \Sigma$. It implies that $p$ divides $|C|$. Therefore, $p^{2}$ divides $|C T|$, which divides $|\mathrm{A}|$, and so we have that $p^{2}$ divides $|A|$, a contradiction. Hence, $C=1$, and $A \leq \operatorname{Aut}(T)$ is almost simple. Since $T \cap X \unlhd X \cong \mathrm{A}_{120}$, it follows that $T \cap X=1$ or $X$. If $T \cap X=1$, then since $\frac{|\mathrm{A}|}{|X|} \mid 2^{4} \cdot 3^{2} \cdot 5^{2}$, we have $|T| \mid 2^{4} \cdot 3^{2} \cdot 5^{2}$; note that $p>5, p|| T \mid$, a contradiction. Thus, $T \cap X=X$, and so $X \leq T$. It follows that $|T: X|$ divides $|A: X|$, which divides $2^{4} \cdot 3^{2} \cdot 5^{2}$. By [18] (pp. 135-136), we can conclude that $T=X \cong A_{120}$. Thus, $A \leq A u t(T) \cong S_{120}$. If $A \cong S_{120}$, then $\left|A_{v}\right|=\frac{|A|}{|G|}=240$, a contradiction to Lemma 3. Hence, $A \cong A_{120}$.

Now assume that the full automorphism group $\mathrm{A}$ is not quasiprimitive on $V \Sigma$. Then there is a minimal normal subgroup $M$ of $A$ that acts nontransitively on $V \Sigma$. Since $M \cap$ $X \unlhd X$, we have $M \cap X=1$ or $X$. For the latter case $M \cap X=X$, we have $X \leq M$, and so $M$ is transitive on $V \Sigma$, a contradiction. For the former case, $M \cap X=1$, then we have that $|M|$ divides $\frac{|\mathrm{A}|}{|X|}$, which divides $2^{4} \cdot 3^{2} \cdot 5^{2}$.

Assume that $M$ is insoluble. Since $|M|$ divides $2^{4} \cdot 3^{2} \cdot 5^{2}$, and the simple groups $A_{5}, A_{6}, \operatorname{PSp}(4,3)$ are the only $\{2,3,5\}$-factor non-abelian simple groups (see [19] (Table 1), and note that the definition of the $\{2,3,5\}$-group is similar to $\{2,3\}$-group); by checking the orders of these groups, it is easy to figure out $M \cong A_{5}$ or $A_{5}^{2}$ or $A_{6}$. Then since $|M| \cdot\left|\mathrm{A}_{120}\right|=|M| \cdot|X|=|L|=|V \Sigma| \cdot\left|L_{v}\right|=\left|\mathrm{A}_{119}\right| \cdot\left|L_{v}\right|$, we have $\left|L_{v}\right|=2^{5} \cdot 3^{2} \cdot 5^{2}$ or $2^{7} \cdot 3^{3} \cdot 5^{3}$ or $2^{6} \cdot 3^{3} \cdot 5^{2}$, a contradiction to the description of the orders of the stabilizers in Lemma 3 .

Assume that $M$ is soluble. Then $M \cong \mathbb{Z}_{2}^{r}$ or $\mathbb{Z}_{3}^{s}$ or $\mathbb{Z}_{5}^{l}$, where $1 \leq r \leq 4,1 \leq s \leq 2$ and $1 \leq l \leq 2$. Let $L=M X$. Then $L=M: X$, a split expansion of $M$ by $X$. Further, we have $L / C_{L}(M) \lesssim \operatorname{Aut}(M) \cong \operatorname{GL}(r, 2)$ or $\operatorname{GL}(s, 3)$ or $\operatorname{GL}(l, 5)$. We note that $M$ is a subgroup of $C_{L}(M)$. If $M=C_{L}(M)$, then we have $L / C_{L}(M)=L / M \cong X \cong A_{120} \lesssim \mathrm{GL}(r, 2)$ or $\mathrm{GL}(s, 3)$ or $\mathrm{GL}(l, 5)$. However, for each $1 \leq r \leq 4,1 \leq s \leq 2$ and $1 \leq l \leq 2, \mathrm{GL}(r, 2)$, $\operatorname{GL}(s, 3)$ or $\operatorname{GL}(l, 5)$ has no subgroup isomorphic to the alternating group $A_{120}$. Hence, we have $M<\mathrm{C}_{L}(M)$ and $1 \neq \mathrm{C}_{L}(M) / M \unlhd L / M \cong \mathrm{A}_{120}$. It implies that $\mathrm{A}_{120} \cong \mathrm{C}_{L}(M) / M$; then $\left|C_{L}(M)\right|=|M| \cdot|X|=|L|$ since $C_{L}(M) \unlhd L$, we have $C_{L}(M)=L=M X$, and $X$ centralizes $M$. Hence, $L=M \times X$. Then $L_{v} / \bar{X}_{v}=L_{v} / L_{v} \cap X \cong L_{v} X / X \cong L / X \cong M$. Thus, $L_{v} \cong X_{v}$.M. Note that with the order of the stabilizers given in Lemma 3 , we conclude $M \cong \mathbb{Z}_{3}$ or $\mathbb{Z}_{5}$. In the case where $M \cong \mathbb{Z}_{3}$, we have $\left|L_{v}\right|=\left|X_{v}\right| \cdot|M|=360$, then $L_{v} \cong \mathrm{A}_{6}$, $A_{6} \cong \operatorname{PGL}(2,5) \cdot \mathbb{Z}_{3}$, but there is no normal subgroup which is isomorphic to PGL $(2,5)$ in $\mathrm{A}_{6}$, a contradiction. In the case where $M \cong \mathbb{Z}_{5}$, we have $\left|L_{v}\right|=\left|X_{v}\right| \cdot|M|=600$, then $L_{v} \cong \mathrm{D}_{10} \times \operatorname{PSL}(2,5), \mathrm{D}_{10} \times \operatorname{PSL}(2,5) \cong \operatorname{PGL}(2,5) . \mathbb{Z}_{5}$; by [17], there is no normal subgroup with order 120 in $\mathrm{D}_{10} \times \operatorname{PSL}(2,5)$, so clearly, $\operatorname{PGL}(2,5) \not \mathrm{D}_{10} \times \operatorname{PSL}(2,5)$, which also leads to a contradiction. This completes the proof of the lemma.

Proof of Theorem 1. Now we are ready to prove our main Theorem 1 . Let $\Sigma=\operatorname{Cos}(X, H, x)$ be the graph as in Construction 1. Then, Lemma 4 shows that $\Sigma$ is a connected 2-arctransitive graph and isomorphic to a nonnormal hexavalent Cayley graph $C a y(G, S)$, with $G \cong A_{119}$. This proves the statement of the former part of Theorem 1 . The next Lemma 5 shows that the full automorphism group Aut $\Sigma$ of the graph $\Sigma$ is isomorphic to alternating group $A_{120}$. This proves the statement of the latter part of Theorem 1 , and so completes the proof of Theorem 1. 
Author Contributions: B.L. (Bo Ling): formal analysis,supervision, W.L.: writing—original draft, B.L. (Bengong Lou): writing-review and editing. All authors have read and agreed to the published version of the manuscript.

Funding: This work was partially supported by the National Natural Science Foundation of China $(12061089,11861076,11701503,11761079)$, and the Natural Science Foundation of Yunnan Province (2019FB139, 2018FB003).

Institutional Review Board Statement: Not applicable.

Informed Consent Statement: Not applicable.

Data Availability Statement: Not applicable.

Conflicts of Interest: The authors declare no potential conflict of interest.

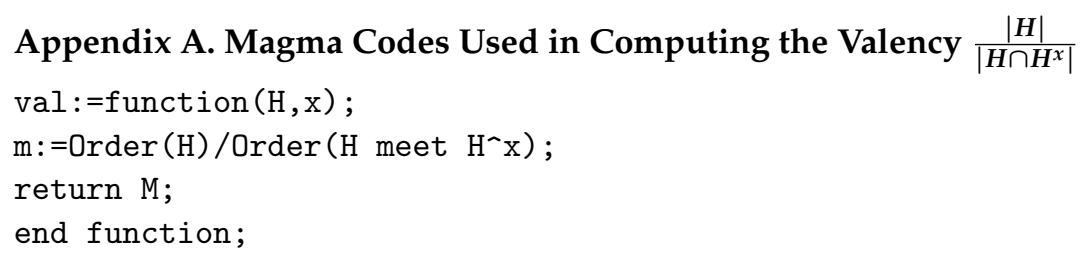

Appendix B. Magma Codes Used in Computing the Elements of $G \cap(H x H)$

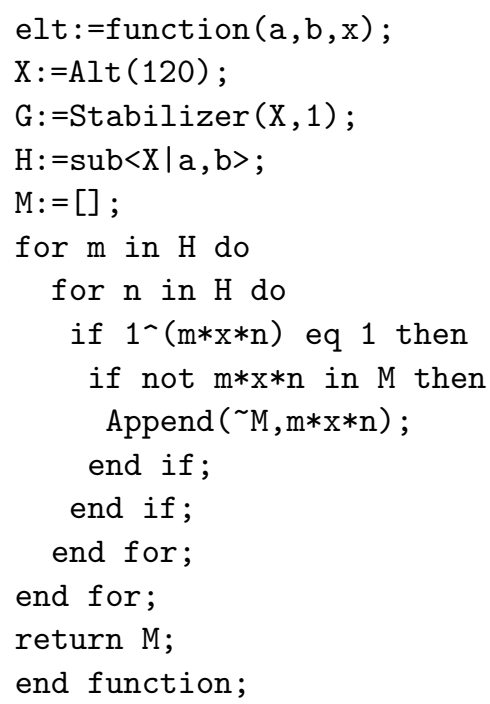

\section{References}

1. Sabidussi, G. On a class of fixed-point-free graphs. Proc. Am. Math. Soc. 1958, 9, 800-804. [CrossRef]

2. Xu, M.Y. Automorphism groups and isomorphisms of Cayley digraphs. Discrete Math. 1998, 182, 309-319. [CrossRef]

3. Heydemann, M.C. Cayley graphs and interconnection networks. In Graph Symmetry; Hahn, G., Sabidussi, G., Eds.; Kluwer Academic Publishing: Dordrecht, The Netherlands, 1997; pp. 167-224.

4. Lakshmivarahan, S.; Jwo, J.S.; Dhall, S.K. Symmetry in interconnection networks based on Cayley graphs of permutation groups: A survey. Parallel Comput. 1993, 19, 361-407. [CrossRef]

5. Fang, X.G.; Ma, X.S.; Wang, J. On locally primitive Cayley graphs of finite simple groups. J. Comb. Theory A 2011, 118, 1039-1051. [CrossRef]

6. Li, C.H. Isomorphisms of Finite Cayley Graphs. Ph.D. Thesis, The University of Western Australia, Perth, Australia, 1996.

7. Xu, S.J.; Fang, X.G.; Wang, J.; Xu, M.Y. On cubic s-arc-transitive Cayley graphs of finite simple groups. Eur. J. Comb. 2005, 26, 133-143. [CrossRef]

8. Xu, S.J.; Fang, X.G.; Wang, J.; Xu, M.Y. 5-arc transitive cubic Cayley graphs on finite simple groups. Eur. J. Comb. 2007, 28, 1023-1036. [CrossRef]

9. Fang, X.G.; Li, C.H.; Xu, M.Y. On edge-transitive Cayley graphs of valency four. Eur. J. Combin. 2004, 25, 1107-1116. [CrossRef]

10. Fang, X.G.; Wang, J.; Zhou, S.M. Tetravalent 2-transitive Cayley graphs of finite simple groups and their automorphism groups. arXiv 2016, arXiv:1611.06308v1.

11. Zhou, J.X.; Feng, Y.Q. On symmetric graphs of valency five. Discret. Math. 2010, 310, 1725-1732. [CrossRef] 
12. Ling, B.; Lou, B.G. A 2-arc transitive pentavalent Cayley graph of A39. Bull. Aust. Math. Soc. 2016, 93, 441-446. [CrossRef]

13. Huppert, B. Eudiche Gruppen I; Springer: Berlin/Heidelberg, Germany, 1967.

14. Sabidussi, B.O. Vertex-transitive graphs. Monash Math. 1964, 68, 426-438. [CrossRef]

15. Lorimer, P. Vertex-transitive graphs of prime valency. J. Graph Theory 1984, 8, 55-68. [CrossRef]

16. Guo, S.T.; Hua, X.H.; Li, Y.T. Hexavalent $(G, s)$-transitive graphs. Czechoslov. Math. J. 2013, 63, 923-931. [CrossRef]

17. Bosma, W.; Cannon, C.; Playoust, C. The MAGMA algebra system I: The user language. J. Symb. Comput. 1997, 24, 235-265. [CrossRef]

18. Gorenstein, D. Finite Simple Groups; Plenum Press: New York, NY, USA, 1982.

19. Huppert, B.; Lempken, W. Simple Groups of Order Divisible by at Most Four Primes; Francisk Skorina Gomel State University: Gomel, Belarus, 2000; Volume 10, pp. 64-75. 